\title{
Estimating Annual Fluctuations in Malaria Transmission Intensity and in the Use of Malaria Control Interventions in Five Sub-Saharan African Countries
}

\author{
RTS,S Epidemiology EPI-MAL-005 Study Group \\ The RTS,S Epidemiology EPI-MAL-005 study group is composed of (per alphabetical order): \\ Elisha Adeniji, ${ }^{1}$ Kwaku Poku Asante, ${ }^{1}$ Owusu Boahen, ${ }^{1}$ Guillaume Compaoré, ${ }^{2}$ Boubacar Coulibaly, ${ }^{2}$ Seyram Kaali, ${ }^{1}$ Youssouf Kabore, ${ }^{3}$ \\ Mathieu Lamy, ${ }^{4 \star}$ John Lusingu, ${ }^{5,6}$ Anangisye Malabeja, ${ }^{5}$ Petra Mens, ${ }^{7}$ Mattéa Orsini, ${ }^{8}$ Lucas Otieno, ${ }^{9}$ Walter Otieno, ${ }^{9}$ Seth Owusu- \\ Agyei, ${ }^{1}$ Janet Oyieko, ${ }^{9}$ Jean-Yves Pirçon, ${ }^{10}$ Nicolas Praet, ${ }^{10}$ François Roman, ${ }^{10}$ Ali Sie, ${ }^{2}$ Valentine Sing'oei, ${ }^{9}$ Sodiomon B. Sirima, ${ }^{3}$ \\ Khadime Sylla, ${ }^{11}$ Roger Tine, ${ }^{11}$ Alfred B. Tiono, ${ }^{3}$ Mathilda Tivura, ${ }^{1}$ Effua Usuf, ${ }^{12}$ and Stéphanie Wéry ${ }^{10} \dagger$ \\ ${ }^{1}$ Kintampo Health Research Centre, Ghana Health Service, Kintampo, Ghana; ${ }^{2}$ Centre de Recherche en Santé de Nouna, Nouna, Burkina Faso; \\ ${ }^{3}$ Centre National de Recherche et de Formation sur le Paludisme (CNRFP), Ouagadougou, Burkina Faso; ${ }^{4}$ Aixial c/o GSK, Wavre, Belgium; \\ ${ }^{5}$ National Institute for Medical Research (NIMR), Korogwe, Tanzania; ${ }^{6}$ University of Copenhagen, Copenhagen, Denmark; ${ }^{7}$ Parasitology Unit, \\ Department of Medical Microbiology, Academic Medical Center, Amsterdam University Medical Centers, University of Amsterdam, Amsterdam, \\ The Netherlands; ${ }^{8} 4$ Clinics clo GSK, Wavre, Belgium; ${ }^{9} \mathrm{KEMRI}$ - Walter Reed Project, US Army Medical Research Directorate-Kenya, Kombewa, \\ Kenya; ${ }^{10}$ GSK, Wavre, Belgium; ${ }^{11}$ Département de Parasitologie, Centre de Recherche de Keur Socé, Faculté de Médecine, Université Cheikh Anta \\ Diop, Dakar, Senegal; ${ }^{12}$ Medical Research Council Unit, The Gambia at London School of Hygiene and Tropical Medicine, Fajara, The Gambia
}

\begin{abstract}
RTS,S/AS01 $\mathrm{E}$ malaria vaccine safety, effectiveness, and impact will be assessed in pre- and post-vaccine introduction studies, comparing the occurrence of malaria cases and adverse events in vaccinated versus unvaccinated children. Because those comparisons may be confounded by potential year-to-year fluctuations in malaria transmission intensity and malaria control intervention usage, the latter should be carefully monitored to adequately adjust the analyses. This observational cross-sectional study is assessing Plasmodium falciparum parasite prevalence (PfPR) and malaria control intervention usage over nine annual surveys performed at peak parasite transmission. Plasmodium falciparum parasite prevalence was measured by microscopy and nucleic acid amplification test (quantitative PCR) in parallel in all participants, and defined as the proportion of infected participants among participants tested. Results of surveys 1 (S1) and 2 (S2), conducted in five sub-Saharan African countries, including some participating in the Malaria Vaccine Implementation Programme (MVIP), are reported herein; 4,208 and 4,199 children were, respectively, included in the analyses. Plasmodium falciparum parasite prevalence estimated using microscopy varied between study sites in both surveys, with the lowest prevalence in Senegalese sites and the highest in Burkina Faso. In sites located in the MVIP areas (Kintampo and Kombewa), PfPR in children aged 6 months to 4 years ranged from $24.8 \%$ to $27.3 \%$, depending on the study site and the survey. Overall, $89.5 \%$ and $86.4 \%$ of children used a bednet in S1 and S2, of whom $68.7 \%$ and $77.9 \%$ used impregnated bednets. No major difference was observed between the two surveys in terms of PfPR or use of malaria control interventions.
\end{abstract}

\section{INTRODUCTION}

Substantial investment to expand existing malaria interventions has resulted in a reduction in the global incidence rate of malaria between 2010 and 2017. ${ }^{1}$ However, between 2015 and 2017, after stagnation, a slight upward trend in malaria incidence was observed. Malaria remains a major cause of death worldwide, with approximately $93 \%$ of all malaria deaths in 2017 occurring in Africa. ${ }^{1}$ To reach the Global Technical Strategy for Malaria 2016-2030 target of reducing global malaria incidence and mortality rates by at least $90 \%$ by $2030,{ }^{2}$ the need for safe and effective malaria vaccines that prevent disease and death and decrease transmission to enable malaria eradication was endorsed and documented in the WHO Malaria Vaccine Technology Roadmap. ${ }^{3}$

GlaxoSmithKline (GSK) has developed, in partnership with the PATH Malaria Vaccine Initiative, a pre-erythrocytic Plasmodium falciparum malaria vaccine, RTS,S/AS01 ${ }_{\mathrm{E}}\left(\mathrm{GSK}^{\mathrm{TM}}\right.$, Wavre, Belgium), for routine immunization of infants and children living in sub-Saharan African (SSA) malaria-endemic countries. In 2015, the European Medicines Agency adopted a positive opinion for the use of the vaccine in children aged 6 weeks to 17 months at the first dose. ${ }^{4}$ In January 2016, the *Address correspondence to Mathieu Lamy, GSK Biologicals, Ave. de
Fleming, 20, Wavre 1300, Belgium. E-mail: mathieu.x.lamy@gsk.com
†At the time of Survey 1, SW was an employee of Keyrus Biopharma c/o GSK.
WHO recommended a pilot implementation of $\mathrm{RTS}, \mathrm{S} / \mathrm{AS} 01_{\mathrm{E}}$ in children as of 5 months of age in three to five epidemiological SSA settings with moderate to high malaria transmission. ${ }^{5}$ In April 2017, the WHO announced the vaccine introduction based on a cluster-randomized design in pilot areas of Ghana, Kenya, and Malawi through the national expanded programs on immunization, in the framework of the Malaria Vaccine Implementation Programme (MVIP). ${ }^{6}$ Today, RTS,S/AS01 ${ }_{E}$ is the first vaccine implemented for the prevention of malaria.

To assess vaccine safety, effectiveness, and impact, GSK designed a pre- and a post-vaccine introduction observational study (Clinical Trials.gov identifiers: NCT02374450 and NCT03855995, respectively), allowing comparison of the occurrence of malaria cases and adverse events in vaccinated versus unvaccinated children. In parallel with those studies, the present observational cross-sectional annual study (NCT02251704) is estimating $P$. falciparum parasite prevalence (PfPR) and the use of malaria control interventions during up to nine consecutive years, applying standardized methodologies and multiple diagnostic testing. More specifically, considering the $\mathrm{WHO}$ recommendation to operate the MVIP in moderate to high transmission areas of SSA, this study will allow 1) characterizing the malaria transmission intensity (MTI) before RTS,S/AS01 ${ }_{E}$ vaccine introduction in different countries/areas, including the ones participating in the MVIP; 2) monitoring overtime fluctuations of MTI and of the use of malaria control interventions before and after vaccine 
introduction in those areas to adjust the pre- and post-vaccine introduction comparison analyses for potential year-to-year and/or geographical variations.

We present here the results of the first two annual surveys that were conducted before vaccine introduction. On completion of all surveys, the data collected in this study involving approximately 50,000 participants representing multiple sites in various SSA countries will provide a unique perspective on malaria prevalence variations across Africa.

\section{MATERIALS AND METHODS}

Ethics. The study was approved by national independent Ethics Committees and local institutional review boards in Burkina Faso (BF), Ghana (GH), Kenya (KE), Senegal (SN), and Tanzania (TZ), and conducted in accordance with the provisions of the International Conference on Harmonisation and Good Clinical Practice guidelines.

Study population. Individuals aged 6 months to $<10$ years, whose parents or legally acceptable representative had provided informed consent for study participation, were randomly selected each year in each of the study sites (see Study design section) using population listings generated from local Health and Demographic Surveillance Systems (HDSS) and following a stratification by age-group (see the Supplemental Appendix Section 1). Children in care, or actively participating in any trial involving the administration of an investigational malaria vaccine and/or drug, were excluded.

Study design. Malaria transmission intensity levels are consensus indicators developed by the Global Malaria Eradication Programme to measure malaria endemicity ${ }^{7}$ using a standardized methodology. There are several methods for estimating MTI, including entomological inoculation rates (EIRs), serological conversion rates (SCRs), and blood parasite prevalence. Although EIR is a standard method, the measure is challenging, and the interpretation and comparability of the setting may be difficult because of vector heterogeneity. ${ }^{8}$ The methodology and interpretation of SCRs to classify the intensity of malaria is still not commonly used. Plasmodium falciparum prevalence, despite requiring trained staff for slide reading, provides a standardized and relatively easy to implement method to assess MTI in study sites of varied transmission intensity, and has often been used in previous epidemiological studies. ${ }^{7,9,10}$ Therefore, PfPR was the selected method to assess potential variations in MTI in the present study.

The study is multicentric with study sites corresponding to geographically limited catchment areas located in low, moderate, or high MTI regions of SSA, and having an HDSS in place.

Up to nine annual cross-sectional surveys will be conducted during the malaria peak transmission period in each study site (from mid-September to mid-December in Western African sites and from mid-April to mid-August in Eastern African sites). To optimize the detection of the peak transmission, each study site has been equipped with a weather station to record meteorological data such as rainfall, temperature, and humidity. Surveys were conducted during the course of the rainy season preferably when rains decrease, which should correspond to the period of highest malaria transmission. In this article, results of the first two annual surveys are presented. Survey 1 (S1) and survey 2 (S2) were conducted in seven study sites in five SSA countries: BF (Nouna, Saponé),
GH (Kintampo), KE (Kombewa), SN (Keur Socé, Niakhar), and TZ (Korogwe). Sites in GH (Kintampo) and KE (Kombewa) are part of the areas where the RTS,S/AS01 $1_{E}$ vaccination will be implemented through the national Expanded Programs on Immunization in the framework of the MVIP. It is important to note that this study is also conducted in SSA areas where the RTS,S/AS01 E vaccine will not be introduced in the framework of the MVIP because GSK initiated the study before the WHO recommendation. ${ }^{5,11}$

Data collection. Demographic details (age and gender), medical history, and information on care-seeking behaviors (hospitalization for malaria within the last 3 months, visits to healthcare provider for fever or malaria treatment in the previous 14 days, anti-malaria therapy received within the last 14 days); malaria control intervention usage (bednets [new \{not older than a year\}, pierced/torn, impregnated], indoor residual spraying [IRS]); the usage of coils, repellents, and local herbs; and malaria potential risk factors (rural/urban area, house construction materials, use of electricity, and open/ closed water source) were recorded for all participants at the time of the survey. Axillary body temperature was measured and recorded during the survey visit.

To assess within-site heterogeneity of PfPR, study areas were mapped by villages using grid referencing and divided into 3-14 segments with a minimum of 10 enrolled individuals per segment. Segments will remain unchanged for the duration of the study.

Assessment of parasitemia. Both microscopy and Nucleic Acid Amplification Tests (NAATs) were used in parallel on all participants to assess parasitemia. The latter are expected to be more sensitive and specific, particularly in cases of low parasite density. ${ }^{12,13}$ In brief, a blood sample was collected by finger prick for thin and thick blood films for the microscopy assessment and filter paper blood spots for NAATs. Blood smears were examined by two local independent microscopists, and any discrepancies were settled by a third reader. Parasitemia was measured by the examination of 100 high-powered fields on thick smear to determine the presence of parasites; in the case of a positive result, additional 100 fields were examined to assess the presence of multiple species. Plasmodium species and sexual forms were identified on thin blood film. Parasite density was computed as the geometric mean of two readings, counting parasites against 200 white blood cells on thin blood film, assuming 8,000 white blood cells/ $\mu \mathrm{L}$. Parasite density was categorized as low $(<2,500$ parasites $/ \mu \mathrm{L})$, medium $(2,500-9,999)$, high $(10,000-19,999)$, or very high $(>20,000)$. In the case of low density (< 10 parasites against 200 leukocytes), parasite count was conducted against 500 white blood cells. The parasite count technique was replicated to count gametocytes. In parallel with microscopy, asexual and sexual parasitemia was assessed by NAATs using both real-time quantitative PCR (QT-PCR) assay and real-time nucleic acid sequence-based amplification (QT-NASBA) assay. Quantitative PCR allowed detection of asexual and sexual parasites combined, the final output being qualitative (positive or negative) and semi-quantitative (high, medium, low, and negative). QT-NASBA allowed detection of gametocytes, the final output being qualitative (positive or negative). Details for blood slide and NAAT assessment of parasitemia are available in the Supplemental Appendix (Sections 2 and 3, respectively). 
If fever (i.e., axillary temperature $\geq 37.5^{\circ} \mathrm{C}$ ) was recorded at the time of the visit or reported to have occurred within 24 hours before the visit, a malaria rapid diagnostic test (RDT) was conducted using blood from the finger prick sampling. If the RDT was positive, then treatment was given according to national guidelines. Moreover, any participant identified as being parasite positive following microscopy was traced to receive treatment according to national guidelines.

Statistical methods. The planned sample size was 600 participants per study site and per survey distributed as 400 participants between the ages of 6 months to 4 years and 200 participants between the ages of 5 and 9 years. The sample size was calculated to ensure sufficiently narrow $\mathrm{Cls}$ around study site-specific PfPR estimates (with a maximum residual standard error of 0.25 ).

Plasmodium falciparum parasite prevalence and prevalence of gametocytes were estimated as the proportion of participants infected, or carrying gametocytes, respectively, among participants tested. Prevalences were estimated by age and by site. The agreement between the two diagnostic methods used in the framework of this study (parasitemia measured by microscopy versus NAATs) was described using the Cohen's kappa coefficient and assessed using the Landis and Koch scale. ${ }^{14}$ The prevalence of Plasmodium species other than $P$. falciparum was estimated as the proportion of infected participants among participants tested. The within-site heterogeneity between segments was tested using Cochran's $Q$ test based on inverse variance weights.

Malaria control intervention coverage was estimated as the proportion of users among participants for which this information was available. The care-seeking behaviors (treatment sought for malaria or fever in the 14 days before the visit and hospitalization for malaria in the last 3 months before the visit) were described as the proportion of participants having sought for health care among all participants. In addition, a risk factor analysis for malaria infection (dependent variable: $P$. falciparum parasitemia as measured by microscopy) was conducted using a multivariable logistic regression with study site as cluster and using a backward strategy for the selection of significant explanatory variables, that is, predefined potential risk factors and/or the use of malaria control interventions (Supplemental Appendix Section 4). Age was computed as a continuous variable.

\section{RESULTS}

Survey 1 and S2 data were collected between October 2014 and August 2015, and between September 2015 and July 2016, respectively. During S1 and S2, 4,215 and 4,204 children were enrolled and 4,208 and 4,199 were included in the analyses (Figure 1), with a balanced distribution across the seven study sites (Supplemental Table 1). Across all sites, $51.3 \%$ of participants in $\mathrm{S} 1$ and $50.3 \%$ in $\mathrm{S} 2$ were males (Supplemental Table 2).

Year-to-year variation in $\boldsymbol{P}$. falciparum prevalence. Plasmodium falciparum parasite prevalence estimated using microscopy varied between study sites in both surveys, with the lowest prevalence figures in Senegalese sites and the highest in BF (Table 1, Figure 2). In Kintampo and Kombewa sites that are located in the MVIP areas, PfPR in children aged 6 months to 4 years ranged from $24.8 \%$ to $27.3 \%$ depending on the study site and the survey. In both surveys and across all sites except in SN, PfPR was lower in the 6-month to < 5-year than in the 5-year to $<10$-year age-group. Plasmodium falciparum parasite prevalence was similar in S1 and S2, except for a higher prevalence in S2 in participants from the 6-month
Survey 1

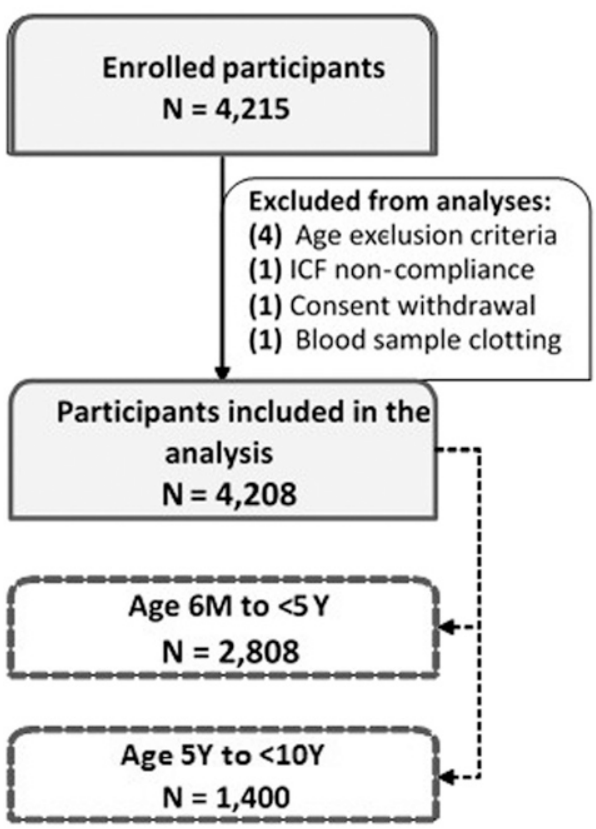

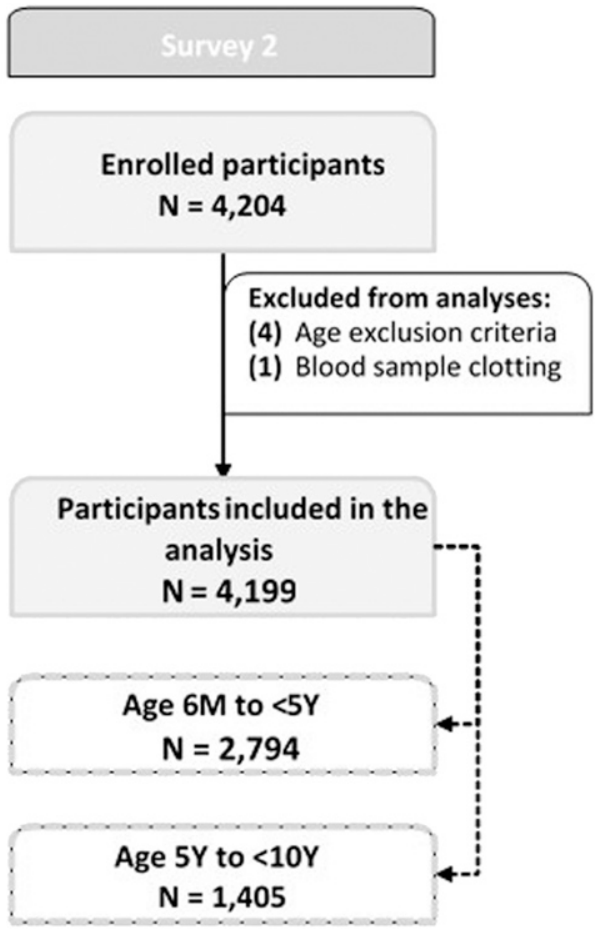

FIGURE 1. Study design overview and study population included in the analysis. ICF $=$ inform consent form; $N=$ number of participants in each group; $6 \mathrm{M}$ to $<5 \mathrm{Y}=6$ months to younger than 5 years; $5 \mathrm{Y}$ to $<10 \mathrm{Y}=5$ years to younger than 10 years. 
TABLE 1

Plasmodium falciparum parasitemia prevalence measured by microscopy by study site and survey according to age-group

\begin{tabular}{|c|c|c|c|c|c|c|c|c|c|}
\hline \multirow[b]{3}{*}{ Age-group } & \multirow[b]{3}{*}{ Study site } & \multicolumn{8}{|c|}{ P. falciparum parasitemia prevalence measured by microscopy slide reading } \\
\hline & & \multicolumn{4}{|c|}{ Survey 1} & \multicolumn{4}{|c|}{ Survey 2} \\
\hline & & $n$ & N & Proportion (\%) & $95 \% \mathrm{Cl}$ & $n$ & N & Proportion (\%) & $95 \% \mathrm{Cl}$ \\
\hline \multirow[t]{7}{*}{$6 \mathrm{M}$ to $<5 \mathrm{Y}$} & Nouna, BF & 249 & 404 & 61.6 & $56.7 ; 66.4$ & 321 & 400 & 80.3 & $76.0 ; 84.0$ \\
\hline & Saponé, BF & 162 & 403 & 40.2 & $35.4 ; 45.2$ & 182 & 399 & 45.6 & $40.7 ; 50.6$ \\
\hline & Kintampo, GH & 99 & 400 & 24.8 & $20.6 ; 29.3$ & 109 & 400 & 27.3 & $22.9 ; 31.9$ \\
\hline & Kombewa, KE & 100 & 402 & 24.9 & $20.7 ; 29.4$ & 108 & 401 & 26.9 & $22.7 ; 31.6$ \\
\hline & Keur Socé, SN & 2 & 400 & 0.5 & $0.1 ; 1.8$ & 4 & 397 & 1.0 & $0.3 ; 2.6$ \\
\hline & Niakhar, SN & 6 & 398 & 1.5 & $0.6 ; 3.3$ & 1 & 397 & 0.3 & $0.01 ; 1.4$ \\
\hline & Korogwe, TZ & 29 & 401 & 7.2 & $4.9 ; 10.2$ & 6 & 400 & 1.5 & $0.6 ; 3.2$ \\
\hline \multirow[t]{7}{*}{5 to $<10 Y$} & Nouna, BF & 154 & 202 & 76.2 & $69.8 ; 81.9$ & 167 & 200 & 83.5 & $77.6 ; 88.4$ \\
\hline & Saponé, BF & 137 & 201 & 68.2 & $61.2 ; 74.5$ & 134 & 200 & 67.0 & $60.0 ; 73.5$ \\
\hline & Kintampo, GH & 115 & 200 & 57.5 & $50.3 ; 64.4$ & 103 & 200 & 51.5 & $44.4 ; 58.6$ \\
\hline & Kombewa, KE & 96 & 197 & 48.7 & $41.6 ; 55.9$ & 81 & 198 & 40.9 & $34.0 ; 48.1$ \\
\hline & Keur Socé, SN & 1 & 200 & 0.5 & $0.01 ; 2.8$ & 2 & 203 & 1.0 & $0.1 ; 3.5$ \\
\hline & Niakhar, SN & 3 & 200 & 1.5 & $0.3 ; 4.3$ & 2 & 204 & 1.0 & $0.1 ; 3.5$ \\
\hline & Korogwe, TZ & 34 & 200 & 17.0 & $12.1 ; 22.9$ & 12 & 200 & 6.0 & $3.1 ; 10.3$ \\
\hline
\end{tabular}

to < 5-year age-group in BF Nouna and lower prevalence in $\mathrm{S} 2$ in both the 6-month to < 5-year and 5-year to < 10-year age-groups in participants from TZ Korogwe. Significant within-site heterogeneity was detected in all sites, except in SN: BF Nouna (S1 $P<0.0001, \mathrm{~S} 2 P=0.0184)$, BF Saponé (S1 $P=0.0321, \mathrm{~S} 2 P<0.0001), \mathrm{TZK}$ Korogwe $(\mathrm{S} 1 P=0.0037, \mathrm{~S} 2 P=$ $0.0275)$, KE Kombewa (S1 $P=0.0220$, S2 $P<0.0001$ ), and $\mathrm{GH}$ Kintampo (S2 only $P=0.0065)$.

Similar trends were observed when measured by QT-PCR, with PfPR varying from $2.7 \%$ in SN Niakhar to $69.9 \%$ in BF Nouna (not measured in SN Keur Socé) in S1 and from 1.3\% in SN Keur Socé to $76.3 \%$ in BF Nouna in S2 (Supplemental Table 3).

The prevalence of gametocytes measured by microscopy ranged from $0.5 \%$ to $4.0 \%$ in S1 and from $0.0 \%$ to $5.3 \%$ in $\mathrm{S} 2$ in all sites, except in BF (15.8\% and $22.7 \%$ in S1 and $12.8 \%$ and $18.2 \%$ in S2 in Nouna and Sapone, respectively). Among participants tested positive for asexual parasites, 7.5-66.7\% carried gametocytes in S1 (Kintampo and Keur Socé, respectively) and $0.0-83.3 \%$ in S2 (Niakhar and Keur Socé, respectively) (Table 2). The proportion of infected participants carrying gametocytes as estimated by QT-NASBA ranged from $13.3 \%$ in SN Niakhar to $59.2 \%$ in BF Sapone in S1 (not measured in Keur Socé) and from $7.1 \%$ in SN Niakhar to $66.7 \%$ in SN Keur Socé in S2 (Supplemental Table 3).

Agreement between diagnostic tests. Across surveys, approximately $21.6 \%$ of participants with a positive QT-PCR result had a negative microscopy reading (285 of 1,268 positive participants per QT-PCR in S1 and 296 of 1,417 in S2) and around $8.8 \%$ of participants positive for microscopy had a negative result with QT-PCR (92 of 1,075 positive participants per microscopy in S1 and 110 of 1,231 in S2) (Table 3). The proportion of participants with a negative result by microscopy among participants with a positive QT-PCR ranged

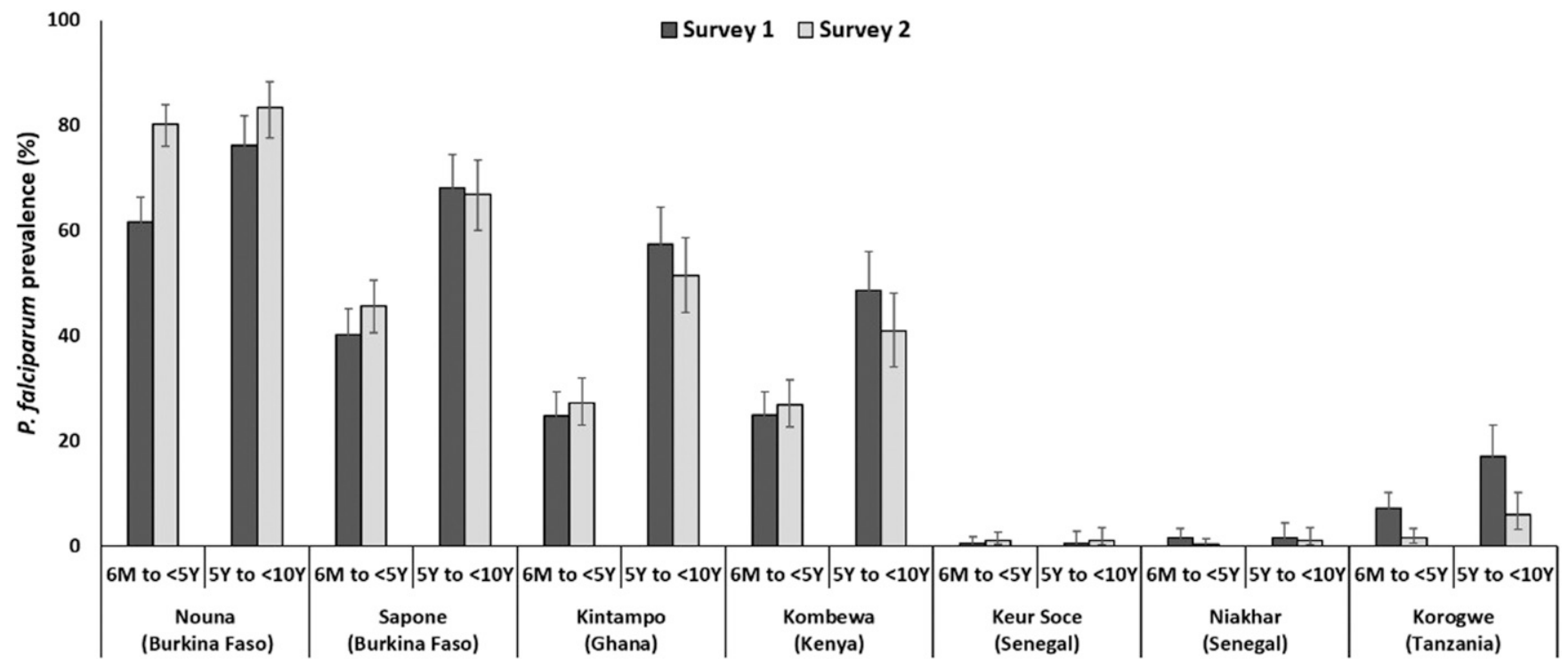

FIGURE 2. Plasmodium falciparum parasitemia prevalence measured by microscopy, by age category, and by site. $6 \mathrm{M}$ to $<5 \mathrm{Y}=6 \mathrm{months}$ to younger than 5 years; $5 \mathrm{Y}$ to $<10 \mathrm{Y}=5$ years to younger than 10 years. Error bars depict $95 \% \mathrm{Cl}$. 
TABLE 2

Gametocyte results measured by microscopy according to $P$. falciparum infection status per study site and survey

\begin{tabular}{|c|c|c|c|c|}
\hline \multirow[b]{3}{*}{ Study site, $n(\%)$} & \multicolumn{4}{|c|}{ Gametocytes measured by microscopy } \\
\hline & \multicolumn{2}{|c|}{$\begin{array}{l}\text { Participants positive for } \\
\text { P. falciparum by microscopy }\end{array}$} & \multicolumn{2}{|c|}{$\begin{array}{l}\text { Participants tested for } \\
\text { P. falciparum by microscopy }\end{array}$} \\
\hline & Survey 1 & Survey 2 & Survey 1 & Survey 2 \\
\hline $\begin{array}{c}\text { Nouna, BF } \\
\text { Positive }\end{array}$ & $\begin{array}{l}N=403 \\
89(22.1)\end{array}$ & $\begin{array}{l}N=488 \\
76(15.6)\end{array}$ & $\begin{array}{l}N=606 \\
96(15.8)\end{array}$ & $\begin{array}{l}N=600 \\
77(12.8)\end{array}$ \\
\hline Saponé, BF & $N=299$ & $N=316$ & $N=604$ & $N=599$ \\
\hline Positive & $111(37.1)$ & $84(26.6)$ & $137(22.7)$ & 109 (18.2) \\
\hline $\begin{array}{l}\text { Kintampo, GH } \\
\text { Positive }\end{array}$ & $\begin{array}{c}N=214 \\
16(7.5)\end{array}$ & $\begin{array}{l}N=212 \\
23(10.9)\end{array}$ & $\begin{array}{l}N=600 \\
18(3.0)\end{array}$ & $\begin{array}{l}N=600 \\
32(5.3)\end{array}$ \\
\hline Kombewa, KE & $N=196$ & $N=189$ & $N=599$ & $N=599$ \\
\hline Positive & $19(9.7)$ & $8(4.2)$ & $24(4.0)$ & $9(1.5)$ \\
\hline $\begin{array}{l}\text { Keur Socé, SN } \\
\text { Positive }\end{array}$ & $\begin{array}{l}N=3 \\
2(66.7)\end{array}$ & $\begin{array}{l}N=6 \\
5(83.3)\end{array}$ & $\begin{array}{c}N=600 \\
5(0.8)\end{array}$ & $\begin{array}{r}N=600 \\
5(0.8)\end{array}$ \\
\hline Niakhar, SN & $N=9$ & $N=3$ & $N=598$ & $N=601$ \\
\hline Positive & $1(11.1)$ & $0.0(0)$ & $3(0.5)$ & $0.0(0)$ \\
\hline $\begin{array}{c}\text { Korogwe, TZ } \\
\text { Positive }\end{array}$ & $N=63$ & $N=18$ & $N=601$ & $N=600$ \\
\hline
\end{tabular}

between $42.9 \%$ and $78.6 \%$ in sites with low MTI (Niakhar, Keur Socé, and Korogwe).

Across all sites, Cohen's kappa coefficient between qualitative results of the two methods of measurement of parasitemia (microscopy versus QT-PCR) using the Landis and Koch scale showed a substantial agreement in both surveys (S1: kappa $=0.75$ [95\% Cl: 0.73; 0.78]; S2: kappa $=0.78$ [95\% Cl: $0.75 ; 0.80])$. Cohen's kappa coefficient between semiquantitative results for parasitemia measured by microscopy versus QT-PCR also showed a substantial agreement in both S1 (kappa $=0.65$ [95\% Cl: 0.63; 0.68]) and S2 (kappa $=0.66$ [95\% Cl: 0.64; 0.68]).

Between 25.0\% (192 of 767 in S2) and 33.8\% (185 of 548 in S1) of participants tested positive for gametocytes by QT-NASBA were also detected positive by microscopy (Supplemental Table 4).

Prevalence of Plasmodium species other than $\boldsymbol{P}$. falciparum. Infection with Plasmodium malariae was observed in $1.5 \%$ of participants in S1 and $3.0 \%$ of participants in S2 (Supplemental Table 5). Coinfection with both $P$. malariae and $P$. falciparum was more frequent than single infection with $P$. malariae alone in both surveys (S1: $4.6 \%$ versus $0.2 \%$; $\mathrm{S} 2: 7.4 \%$ versus $1.2 \%)$. Of the 62 participants in $S 1$ and 128 participants in S2 infected with P. malariae, 55 (88.7\%) and 91 (71.1\%) were also infected with $P$. falciparum, respectively. Infection with Plasmodium ovale was low in both $\mathrm{S} 1$ and $\mathrm{S} 2(0.5 \%$ and $0.2 \%$ of participants). Plasmodium vivax was not observed in S1 and in only one participant in S2. Infections with species other than $P$. falciparum were mostly observed in sites of medium-to-high $P$. falciparum prevalence.

Year-to-year variation in the use of malaria control interventions. Overall, $89.5 \%$ and $86.4 \%$ of children used a bednet the night before the survey in S1 and S2, respectively (Table 4, Supplemental Table 6). The highest use of bednets was in KE Kombewa (97.5\%) in S1 and TZ Korogwe (99.2\%) in S2, and the lowest in GH Kintampo (70.2\%) in S1 and SN Niakhar $(69.6 \%)$ in S2 (Table 4). A decrease in usage between the two surveys was observed in Kombewa, Keur Socé, and Niakhar, whereas an increase was observed in Kintampo and Korogwe.
Overall, $70-80 \%$ of the bednets were impregnated, $60-70 \%$ were new, and approximately $25 \%$ were torn (Supplemental Table 6). Details for the characterization of bednet usage (new, impregnated, and pierced/torn) by study site are shown in Figure 3.

Participant's recall of IRS in the past 12 months was recorded for a very low number of participants (across surveys, $4.1 \%$ overall), mainly in SN (Supplemental Table 7 ).

Overall, usage of coils and repellents was limited, around $10 \%$ of the population in both surveys with variations per site between $1 \%$ and $40 \%$ according to the survey (Supplemental Table 7).

Year-to-year variation in reported fever and care-seeking behaviors. Fever in the last 24 hours was reported for approximately a quarter of the participants in both surveys, with differences across study sites ranging from $3.6 \%$ in BF Saponé to $63.8 \%$ in KE Kombewa in S1 and from 3.0\% in SN Keur Socé to $71.6 \%$ in KE Kombewa in S2 (Supplemental Table 8). Across all sites, occurrence of fever was higher in $P$. falciparum-infected versus non-infected participants (35.6\% versus $21.1 \%$ in S1 and $33.5 \%$ versus $22.5 \%$ in S2, respectively). In both surveys, fever was more frequently reported by participants with higher parasite densities (Supplemental Table 9).

In S1, 15.7\% of participants had sought treatment against malaria or fever in the 14 days before the survey compared with $12.8 \%$ in S2, ranging from $0.0 \%$ to up to $33.3 \%$ depending on the study site and on the survey (Table 5, Supplemental Table 10). Plasmodium falciparum-infected children sought treatment against malaria or fever more often than non-infected children $(20.1 \%$ versus $14.0 \%$ in $\mathrm{S} 1$ and $20.0 \%$ versus $9.8 \%$ in $\mathrm{S} 2$, respectively).

The proportion of participants hospitalized for malaria was $2.6 \%$ in $\mathrm{S} 1$ and $2.8 \%$ in $\mathrm{S} 2$, with no marked difference between $P$. falciparum-infected and non-infected participants $2.8 \%$ versus $2.5 \%$ in $\mathrm{S} 1$ and $3.4 \%$ versus $2.6 \%$ in S2, respectively; (Table 5, Supplemental Table 10).

Association between potential risk factors and $\boldsymbol{P}$. falciparum infection. An exploratory multivariable model was used to assess the association between potential risk factors or malaria control interventions and $P$. falciparum infection. Across both surveys, houses equipped with electricity (odds ratio [OR]: S1 0.75 [95\% Cl: 0.61; 0.93]; S2 0.89 [95\% Cl: 0.80; 0.98]), cement/plaster walls versus mud (OR: S1 0.81 [95\% Cl: 0.69; 0.96]; S2 0.87 [95\% Cl: 0.76; 0.99]), and nets on all windows (OR: S1 0.73 [95\% Cl: 0.63; 0.84]; S2 0.79 [95\% Cl: $0.66 ; 0.94]$ ) were associated with a lower risk of infection with $P$. falciparum. In addition, older age was associated with a higher risk of infection (OR: 1.14 [95\% Cl: 1.05; 1.24] in S1 and 1.09 [95\% Cl: 1.02; 1.16] in S2; Supplemental Tables 11 and 12).

Figure 4 represents a plain language summary, which elaborates on the epidemiologic study relevance that could be shared with patients by healthcare professionals.

\section{DISCUSSION}

Characterizing MTI in different SSA settings. Using PfPR as a proxy, this study aims at characterizing $\mathrm{MTI}^{15}$ in different SSA settings, including areas in GH and KE where the RTS,S/ $\mathrm{ASO1}_{\mathrm{E}}$ malaria vaccine is currently introduced in the framework of the MVIP. More specifically, considering the WHO 
TABLE 3

Agreement between microscopy and QT-PCR test results by survey

\begin{tabular}{|c|c|c|c|c|c|c|c|c|c|c|c|c|}
\hline \multirow[b]{4}{*}{ Study site } & \multirow{4}{*}{$\begin{array}{l}\text { P. falciparum } \\
\text { measured by } \\
\text { microscopy }\end{array}$} & & \multicolumn{5}{|c|}{ Survey 1} & \multicolumn{5}{|c|}{ Survey 2} \\
\hline & & & \multicolumn{5}{|c|}{ QT-PCR } & \multicolumn{5}{|c|}{ QT-PCR } \\
\hline & & & \multicolumn{2}{|c|}{ Positive $(N=1,268)$} & \multicolumn{2}{|c|}{ Negative $(N=2,069)$} & \multirow{2}{*}{$\begin{array}{c}\begin{array}{c}\text { Total } \\
N=3,337\end{array}\end{array}$} & \multicolumn{2}{|c|}{ Positive $(N=1,417)$} & \multicolumn{2}{|c|}{ Negative $(N=2,737)$} & \multirow{2}{*}{$\begin{array}{c}\text { Total } \\
(N=4,154)\end{array}$} \\
\hline & & & $n$ & $\%$ & $n$ & $\%$ & & $n$ & $\%$ & $n$ & $\%$ & \\
\hline \multirow[t]{4}{*}{ Nouna, BF } & Positive & $\begin{array}{l}n \\
\%\end{array}$ & $\begin{array}{l}347 \\
85.5\end{array}$ & $\begin{array}{c}90.1 \\
-\end{array}$ & $\begin{array}{l}38 \\
21.7\end{array}$ & $\begin{array}{l}9.9 \\
-\end{array}$ & 385 & $\begin{array}{l}400 \\
87.5\end{array}$ & $\begin{array}{c}82.1 \\
-\end{array}$ & $\begin{array}{l}87 \\
61.3\end{array}$ & $\begin{array}{c}17.9 \\
-\end{array}$ & 487 \\
\hline & Negative & $n$ & 59 & 30.1 & 137 & 69.9 & 196 & 57 & 50.9 & 55 & 49.1 & 112 \\
\hline & & $\%$ & 14.5 & - & 78.3 & - & & 12.5 & - & 38.7 & - & \\
\hline & Total & $n$ & 406 & - & 175 & - & & 457 & - & 142 & - & - \\
\hline \multirow[t]{5}{*}{ Saponé, BF } & Positive & $n$ & 212 & 99.1 & 2 & 0.9 & 214 & 312 & 98.7 & 4 & 1.3 & 316 \\
\hline & & $\%$ & 86.2 & - & 1.1 & - & & 80.0 & - & 1.9 & - & \\
\hline & Negative & $n$ & 34 & 16.0 & 178 & 84.0 & 212 & 78 & 27.6 & 205 & 72.4 & 283 \\
\hline & & $\%$ & 13.8 & - & 98.9 & - & & 20.0 & - & 98.1 & - & - \\
\hline & Total & $n$ & 246 & - & 180 & - & - & 390 & - & 209 & - & - \\
\hline \multirow[t]{5}{*}{ Kintampo, GH } & Positive & $n$ & 188 & 90.0 & 21 & 10 & 209 & 201 & 94.8 & 11 & 5.2 & 212 \\
\hline & & $\%$ & 81.4 & - & 6.3 & - & & 71.5 & & 3.4 & - & \\
\hline & Negative & $n$ & 43 & 12.1 & 313 & 87.9 & 356 & 80 & 20.6 & 308 & 79.4 & 388 \\
\hline & & $\%$ & 18.6 & - & 93.7 & - & & 28.5 & & 96.6 & - & \\
\hline & Total & $n$ & 231 & - & 334 & - & - & 281 & - & 319 & - & - \\
\hline \multirow[t]{5}{*}{ Kombewa, KE } & Positive & $n$ & 174 & 88.8 & 22 & 11.2 & 196 & 185 & 97.9 & 4 & 2.1 & 189 \\
\hline & & $\%$ & 66.4 & - & 6.5 & - & & 78.4 & - & 1.1 & - & \\
\hline & Negative & $n$ & 88 & 21.8 & 315 & 78.2 & 403 & 51 & 12.4 & 359 & 87.6 & 410 \\
\hline & & $\%$ & 33.6 & - & 93.5 & - & & 21.6 & & 98.9 & - & \\
\hline & Total & $n$ & 262 & - & 337 & - & - & 236 & & 363 & - & - \\
\hline \multirow[t]{5}{*}{ Keur Socé, SN } & Positive & $n$ & - & - & - & - & - & 4 & 66.7 & 2 & 33.3 & 6 \\
\hline & & $\%$ & - & - & - & - & & 57.1 & & 0.4 & - & \\
\hline & Negative & $n$ & - & - & - & - & - & 3 & 0.5 & 547 & 99.5 & 550 \\
\hline & & $\%$ & - & - & - & - & & 42.9 & - & 99.6 & - & \\
\hline & Total & $n$ & - & & - & - & - & 7 & - & 549 & - & - \\
\hline \multirow[t]{5}{*}{ Niakhar, SN } & Positive & $n$ & 7 & 87.5 & 1 & 12.5 & 8 & 3 & 100.0 & 0 & 0.0 & 3 \\
\hline & & $\%$ & 46.7 & - & 0.2 & & & 21.4 & - & 0.0 & - & - \\
\hline & Negative & $n$ & 8 & 1.4 & 549 & 98.6 & 557 & 11 & 1.8 & 587 & 98.2 & 598 \\
\hline & & $\%$ & 53.3 & - & 99.8 & - & & 78.6 & - & 100.0 & - & - \\
\hline & Total & $n$ & 15 & - & 550 & - & - & 14 & - & 587 & - & - \\
\hline \multirow[t]{5}{*}{ Korogwe, TZ } & Positive & $n$ & 55 & 87.3 & 8 & 12.7 & 63 & 16 & 88.9 & 2 & 11.1 & 18 \\
\hline & & $\%$ & 50.9 & - & 1.6 & - & & 50.0 & - & 0.4 & - & - \\
\hline & Negative & $n$ & 53 & 9.9 & 485 & 90.1 & 538 & 16 & 2.7 & 566 & 97.3 & 582 \\
\hline & & $\%$ & 49.1 & - & 98.4 & - & & 50.0 & - & 99.6 & - & - \\
\hline & Total & $n$ & 108 & - & 493 & - & - & 32 & - & 568 & - & - \\
\hline \multirow[t]{4}{*}{ Overall total } & Positive & $\begin{array}{l}n \\
\%\end{array}$ & $\begin{array}{l}983 \\
77.5\end{array}$ & 91.4 & $\begin{array}{r}92 \\
4.4\end{array}$ & $\begin{array}{l}8.6 \\
-\end{array}$ & 1,075 & $\begin{array}{r}1,121 \\
79.1\end{array}$ & 91.1 & $\begin{array}{r}110 \\
4.0\end{array}$ & $\begin{array}{l}8.9 \\
-\end{array}$ & 1,231 \\
\hline & Negative & $n$ & 285 & 12.6 & 1,977 & 87.4 & 2,262 & 296 & 10.1 & 2,627 & 89.9 & 2,923 \\
\hline & & $\%$ & 22.5 & - & 95.6 & - & & 20.9 & - & 96.0 & - & \\
\hline & Total & $n$ & 1,268 & - & 2,069 & - & - & 1,417 & - & 2,737 & - & - \\
\hline
\end{tabular}

$\mathrm{PCR} ; \%=$ percentage of participants with available results.

recommendation to operate the MVIP in moderate to high transmission areas of SSA, PfPR in children aged 6 months to 4 years in Kintampo and Kombewa sites was high, ranging

TABLE 4

Bednet usage the night before the survey by study site and survey

\begin{tabular}{|c|c|c|c|c|}
\hline \multirow[b]{3}{*}{ Site, country } & \multicolumn{4}{|c|}{ Bednet usage the night before the survey } \\
\hline & \multicolumn{2}{|c|}{ Survey $1(N=4,208)$} & \multicolumn{2}{|c|}{ Survey $2(N=4,199)$} \\
\hline & $N$ & $\%(95 \% \mathrm{Cl})$ & $n$ & $\%(95 \% \mathrm{Cl})$ \\
\hline Nouna, BF & 547 & $90.3(87.6 ; 92.5)$ & 530 & $88.3(85.5 ; 90.8)$ \\
\hline Saponé, BF & 557 & $92.2(89.8 ; 94.2)$ & 534 & $89.1(86.4 ; 91.5)$ \\
\hline Kintampo, GH & 421 & $70.2(66.3 ; 73.8)$ & 497 & $82.8(79.6 ; 85.8)$ \\
\hline Kombewa, KE & 584 & $97.5(95.9 ; 98.6)$ & 534 & $89.1(86.4 ; 91.5)$ \\
\hline Keur Socé, SN & 568 & 94.7 (92.6; 96.3) & 520 & 86.7 (83.7; 89.3) \\
\hline Niakhar, SN & 547 & $91.5(88.9 ; 93.6)$ & 418 & $69.6(65.7 ; 73.2)$ \\
\hline Korogwe, TZ & 544 & $90.5(87.9 ; 92.7)$ & 595 & $99.2(98.1 ; 99.7)$ \\
\hline Overall & 3,768 & $89.5(88.6 ; 90.5)$ & 3,628 & $86.4(85.3 ; 87.4)$ \\
\hline
\end{tabular}

from $24.8 \%$ to $27.3 \%$ depending on the study site and the survey. Similar PfPR was estimated by Drakeley et al., ${ }^{9}$ indicating a stable mesoendemic MTI level (PfPR $=10-50 \%)$ in those areas. ${ }^{10,16}$ Plasmodium falciparum parasite prevalence varied largely between sites as it was expected from various preselected transmission intensity areas. In both surveys, the two sites in BF had the highest PfPR and the two sites in SN, the lowest. Plasmodium falciparum parasite prevalence rates recorded in this study are in line with previous findings. ${ }^{9,16-18}$

Across most sites, the PfPR was lower in the 6-month to $<5$-year than in the 5-year to < 10-year age-group. In addition, a risk factor analysis highlighted an association between older age and higher risk of infection. Those results corroborate the findings of other studies, identifying increasing age as a risk factor for carrying malaria blood stage parasites. ${ }^{9,16,19}$ This may potentially be explained by the fact that younger children benefit from a more focused usage of control interventions (bednets). ${ }^{20}$ Another explanation would be increased immunity in older children due to repeated exposure to the parasite, 
■New bed net $\quad$ Impregnated bed net $\quad$ Pierced/torn bed net

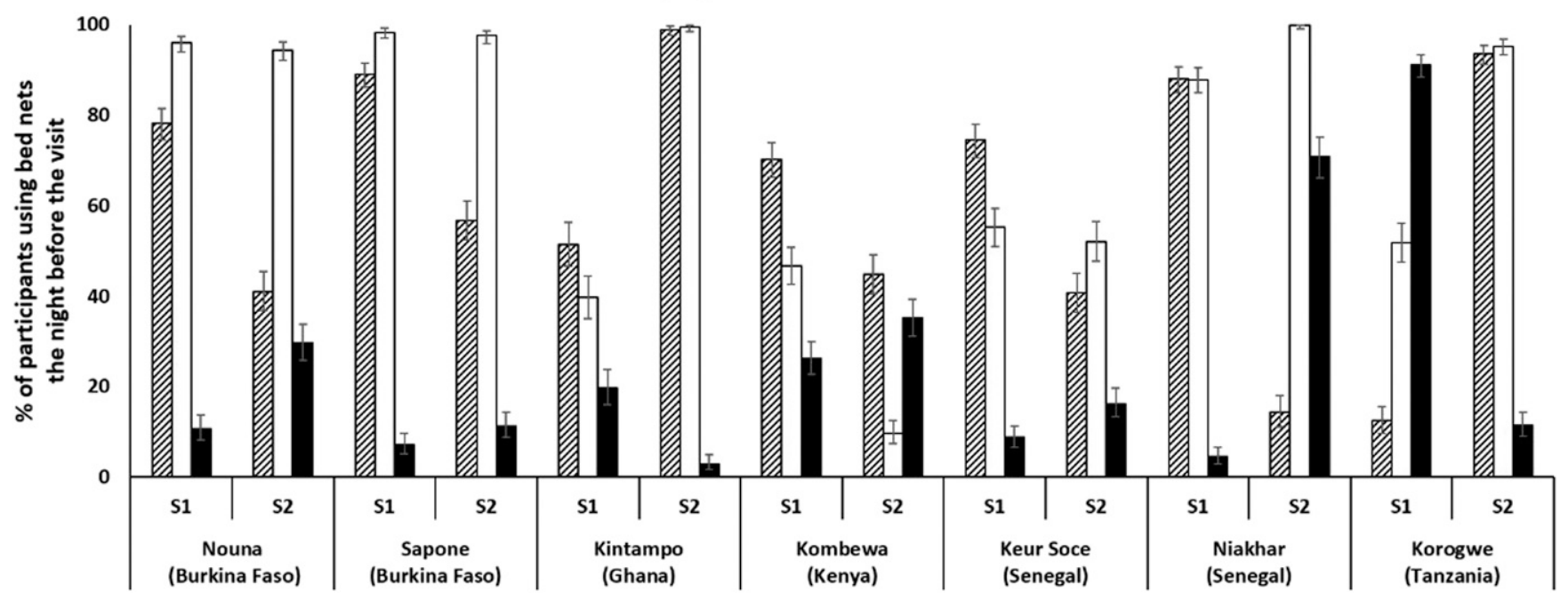

FIGURE 3. Bednet characterization according to obsolescence, impregnation, and condition, by survey and by site. impregnated bednet $=$ bednet dipped in an insecticide liquid before or after purchase; new bednet = bednet not older than 1 year; S1 = survey $1 ; \mathrm{S} 2=$ survey 2 . Error bars depict $95 \% \mathrm{Cl}$.

leading to asymptomatic carriage and a lower probability to be treated than in symptomatic children. ${ }^{21,22}$

Infection with $P$. malariae was observed in a few participants, and infection with $P$. ovale was rare, which supports observations from other studies conducted across SSA. ${ }^{23-26}$ Across all sites and surveys, only one Plasmodium vivax infection was identified by microscopy in TZ, which differs from Twohig et al.' ${ }^{27}$ recent findings of growing evidence of this species in SSA. A high proportion of coinfections with $P$. falciparum was observed in P. malariae-infected participants (between $70 \%$ and $90 \%$ ) and $P$. ovale-infected participants (around 60\%). Similarly, high percentages of $P$. falciparum coinfection in $P$. malariae-infected participants was previously reported in Guinea (97\%), ${ }^{23}$ Uganda $(91 \%),{ }^{24}$ and in the Democratic Republic of the Congo (90\%), ${ }^{25}$ with lower percentages reported in rural BF $(67 \%)^{26}$ and Benin $(34 \%){ }^{23}$

Approximately $20 \%$ of participants positive for $P$. falciparum by QT-PCR were undetected by microscopy. Classifying the study sites by MTI, this proportion was higher in low MTI sites. This might be explained by a relatively lower sensitivity of microscopy readings than nucleic acid related techniques, particularly in low parasite density infections, which are more frequently observed in low MTI areas. ${ }^{12,13}$ Nevertheless, the kappa statistic estimated a substantial agreement between microscopy and NAAT techniques using either qualitative or semi-quantitative real-time PCR.

Monitoring year-to-year variations in MTI and in the use of malaria control interventions. In addition, the present results are establishing a standardized baseline for further estimation of the year-to-year variations in PfPR and in the use of malaria control interventions, which are key variables to be monitored before and after vaccine introduction in the MVIP areas. Little variation in $P$. falciparum prevalence between the first two annual surveys was observed among most sites. Usage of bednets as a malaria control intervention was high in all sites and in both surveys (ranging 70-99\%). Some variation in the usage of bednets was observed in all sites, except in BF.

In S2, participants not using mosquito coils, insecticide sprays, or repellents against malaria vectors were significantly less likely to be infected with $P$. falciparum (OR: S2 0.88 [95\% Cl: 0.782 ; 0.988]); however, no such association was observed in $\mathrm{S} 1$. Various entomological studies have questioned the efficacy of repellents and coils as effective malaria prevention measures and highlighted the false sense of protection perceived by the user. ${ }^{28-30}$ Our data do not allow to draw robust conclusions at this stage, and the trend observed in S2 should be closely monitored in the subsequent cross-sectional surveys.

Study limitations. As with all interview-questionnairebased studies, this study could have been subject to information or recall bias as data related to bednet usage, control interventions usage, and care-seeking behaviors were collected from parents' recollection as opposed to objective observation. The impact of these potential information biases on the study results is estimated to be limited because of the proximity in time between the occurrence of the event for

TABLE 5

Care-seeking behaviors (treatment sought for fever and hospitalization for malaria) according to $P$. falciparum infection status by microscopy and survey

\begin{tabular}{|c|c|c|c|c|c|c|c|c|c|c|}
\hline \multirow[b]{2}{*}{ Care-seeking behavior } & \multirow[b]{2}{*}{ Survey } & \multicolumn{3}{|c|}{ P. falciparum infected } & \multicolumn{3}{|c|}{ P. falciparum not infected } & \multicolumn{3}{|c|}{ Total } \\
\hline & & $N$ & $N$ & $\%(95 \% \mathrm{Cl})$ & $n$ & $N$ & $\%(95 \% \mathrm{Cl})$ & $n$ & $N$ & $\%(95 \% \mathrm{Cl})$ \\
\hline Treatment sought for malaria or & S1 & 238 & 1,187 & $20.1(17.8 ; 22.4)$ & 424 & 3,021 & $14.0(12.8 ; 15.3)$ & 662 & 4,208 & $15.7(14.6 ; 16.9)$ \\
\hline fever in the previous 14 days & S2 & 247 & 1,232 & $20.0(17.8 ; 22.4)$ & 290 & 2,967 & $9.8(8.7 ; 10.9)$ & 537 & 4,199 & $12.8(11.8 ; 13.8)$ \\
\hline Hospitalization for malaria in the & S1 & 33 & 1,187 & $2.8(1.9 ; 3.9)$ & 76 & 3,021 & $2.5(2.0 ; 3.1)$ & 109 & 4,208 & $2.6(2.1 ; 3.1)$ \\
\hline past 3 months & S2 & 42 & 1,232 & $3.4(2.5 ; 4.6)$ & 77 & 2,967 & $2.6(2.1 ; 3.2)$ & 119 & 4,199 & $2.8(2.4 ; 3.4)$ \\
\hline
\end{tabular}




\section{Plain Language Summary}

\section{What is the context?}

- Malaria is a major health issue in Africa with more than 400,000 deaths each year, mainly among young children. The deadly form of the disease is mostly caused by a parasite called Plasmodium falciparum transmitted by mosquitoes.

- Environmental and climatic factors, like rainfalls, influence mosquitoes' proliferation. The use of malaria control interventions (such as mosquito bednets) affects the number of malaria infections in humans.

- A malaria vaccine, RTS,S/AS01, developed by GSK has been introduced in 2019 in selected areas of 3 African countries as a pilot implementation programme coordinated by the World Health Organization. Following the variation of the proportion of malaria-infected people over time is an important factor to help assess vaccine impact on malaria incidence.

\section{What is new?}

- The entire study will include approximately 50,000 children over a 9 years span, conducted before and after vaccine implementation to evaluate changes over time. We present here the results of the first two surveys conducted during the first and second years before vaccine implementation.

- We have assessed the prevalence of Plasmodium falciparum parasites in the blood of children up to 10 years old and have monitored the usage of malaria control interventions and care-seeking behaviours.

- The prevalence of Plasmodium falciparum measured during the peak transmission season, varied largely between countries (less than $2 \%$ in Senegal and over $50 \%$ in Burkina Faso), but it was consistent with literature data.

- No major difference was observed between the 2 surveys in the malaria transmission intensity or use of malaria control interventions, but more surveys are needed to confirm this trend.

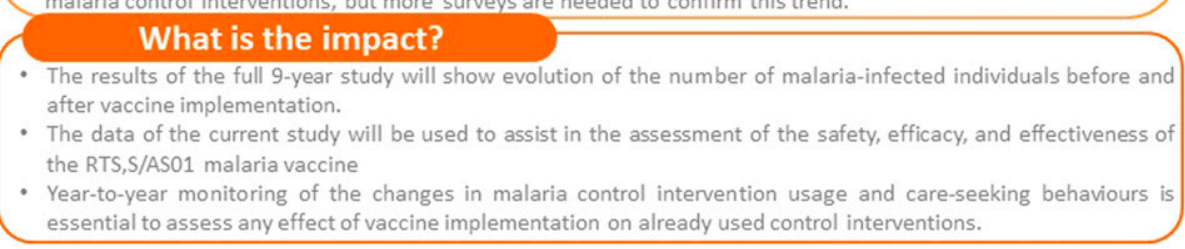

FIGURE 4. Plain language summary. This figure appears in color at www.ajtmh.org.

which information is collected and the interview itself. Another study limitation may be related to both sensitivity and specificity of microscopy slide readings. Slide reading performances may indeed vary depending on the parasite density and species identification. However, the kappa statistic estimated a substantial agreement between microscopy and QTPCR, the latter being able to detect a higher number of low density infections than microscopy and RDT. ${ }^{12,31}$ Moreover, this should mainly impact low transmission settings due to the high proportion of low-density infections.

\section{CONCLUSION}

The present article summarizes the results of the first two annual surveys of a larger malaria prevalence study aiming at characterizing MTI in light of the use of malaria control interventions and other environmental factors. Our results confirm that the high PfPR observed in study sites that are part of the MVIP is in line with the WHO recommendation to operate the program in moderate to high transmission areas. In addition, our results are key to inform on the potential occurrence of annual fluctuations in MTI that may influence the assessment of the RTS,S/AS01 $\mathrm{E}$ vaccine safety, effectiveness, and impact. The observations based on the first two surveys of this study do not indicate major temporal changes in terms of Plasmodium prevalence or use of malaria control interventions, but more surveys are needed to confirm this trend. The data generated in this study will be used to create variables to adjust the temporal and concurrent comparison analyses of the RTS,S/AS01 $1_{E}$ vaccine safety, impact, and effectiveness study. More specifically, the year- and site-specific PfPR computed on unvaccinated study participants will be included as covariates in the regression models of the safety, impact, and effectiveness study to assess annual fluctuations and/or changes due to other malaria control interventions.

To a broader extent, the large sample size of this study (approximately 50,000 participants during the course of 9 years) and the use of a standardized methodology across multiple sites in various countries in SSA using multiple testing will provide a unique perspective on malaria prevalence variations across Africa.

Received October 25, 2019. Accepted for publication August 7, 2020. Published online September 21, 2020.

Note: Supplemental Appendix and tables appear at www.ajtmh.org.

Acknowledgments: We thank the children, their families, and their communities who participated in this study; the investigators, and their clinical and laboratory teams for their contribution to the study and their support and care of participants; and also thank Shyam Prasad TR (TCS c/o GSK) for data management, Aparna Yarraguntla for statistical analysis (ICON, c/o GSK), Roland Vaudry (GSK) and William Zonta (Modis, Belgium c/o GSK) for publication management, and Sarah Benns (Independent medical writer on behalf of GSK) for drafting the manuscript.

Financial support: This trial was sponsored and funded by GlaxoSmithKline Biologicals SA (GSK). GSK also funded all costs associated with the development and the publishing of the present manuscript.

Disclosure: F. R., J.-Y. P., and S. W. are employees of the GSK group of companies. At the time of the study, N. P. was an employee of the 
GSK group of companies. F. R., J.-Y. P., and N. P. hold shares in the GSK group of companies. A. M., A. S., A. B. T., B. C., E. A., G. C., J. L., J. O., K. P. A., K. S., L. O., M. T., O. B., P. M., R. T., S. K., S. O.-A., S. B. S., V.S., W. O., and Y. K., via their institutions, received grants from the GSK group of companies for the conduct of this study/work. A. S., A. B. T., B. C., E. A., G. C., J. O., K. P. A., L. O., M. T., O. B., S. K., S. O.-A., S. B. S., V. S., W. O., and Y. K., via their institutions, received grants from the GSK group of companies for the conduct of studies outside the submitted work. L. O. report grants from GSK Trust in Science outside the submitted work. M. L. is an employee of Aixial on behalf of the GSK group of companies. M. O. is an employee of 4 Clinics on behalf of the GSK group of companies. At the time of the study, E. U. was a consultant for the GSK group of companies. GSK makes available anonymized individual participant data and associated documents from interventional clinical studies which evaluate medicines, upon approval of proposals, submitted to www.clinicalstudydatarequest.com. To access data for other types of GSK sponsored research, for study documents without patientlevel data, and for clinical studies not listed, please submit an enquiry via the website. All authors reviewed and commented on a draft version of the manuscript and gave their final approval for it to be submitted for publication.

Authors' addresses: Elisha Adeniji, Kwaku Poku Asante, Owusu Boahen, Seyram Kaali, Seth Owusu-Agyei, and Mathilda Tivura, Kintampo Health Research Centre, Ghana Health Service, Accra, Ghana, E-mails: elisha.adeniji@kintampo-hrc.org, kwakupoku.asante@kintampohrc.org, owusu.boahen@kintampo-hrc.org, kaali.seyram@kintampo-hrc. org, sowusuagyei@uhas.edu.gh, and mathilda.tivura@kintampohrc.org. Guillaume Compaoré, Boubacar Coulibaly, and Ali Sie, Centre de Recherche en Santé de Nouna, Nouna, Burkina Faso, E-mails: guidedeo@yahoo.fr, boubacar@fasonet.bf, and alisie.crsn@ fasonet.bf. Youssouf Kabore, Sodiomon B. Sirima, and Alfred B. Tiono, Centre National de Recherche et de Formation sur le Paludisme (CNRFP), Ouagadougou, Burkina Faso, E-mails: kabyouf@ yahoo.fr, s.sirima.cnlp@fasonet.bf, and t.alfred@fasonet.bf. Mathieu Lamy, Aixial c/o GSK, Wavre, Belgium, E-mail: mathieu.x.lamy@ gsk.com. John Lusingu, National Institute for Medical Research (NIMR), Korogwe, Tanzania, and University of Copenhagen, Copenhagen, Denmark, E-mail: jpalusingu@yahoo.co.uk. Anangisye Malabeja, National Institute for Medical Research (NIMR), Korogwe, Tanzania, E-mail: malabeja1@yahoo.com. Petra Mens, Parasitology Unit, Department of Medical Microbiology, Academic Medical Center, Amsterdam University Medical Centers, University of Amsterdam, Amsterdam, The Netherlands, E-mail: p.f.mens@amc.uva.nl. Mattéa Orsini, 4Clinics c/o GSK, Wavre, Belgium, E-mail: mattea.x.orsini@ gsk.com. Lucas Otieno, Walter Otieno, Janet Oyieko, and Valentine Sing'oei, KEMRI - Walter Reed Project, US Army Medical Research Directorate-Kenya, Kombewa, Kenya, E-mails: lucas.tina@usamruk.org, walter.otieno@usamru-k.org, janet.oyieko@usamru-k.org, and valentine.singoei@usamru-k.org. Jean-Yves Pirçon, François Roman, and Stéphanie Wéry, GSK, Wavre, Belgium, E-mails: jean-yves.x. pircon@gsk.com, francois.p.roman@gsk.com, and stephanie.x.wery@ gsk.com. Nicolas Praet, GSK, Wavre, Belgium, and Janssen Pharmaceutica NV, Beerse, Belgium, E-mail: nicolas.x.praet@gsk.com. Khadime Sylla and Roger Tine, Faculté de Médecine, Département de Parasitologie, Centre de Recherche de Keur Socé, Université Cheikh Anta Diop, Dakar, Senegal, E-mails: khasylla@gmail.com and rogertine@hotmail.com. Effua Usuf, Medical Research Council Unit, The Gambia at London School of Hygiene and Tropical Medicine, Fajara, The Gambia, E-mail: effuau@gmail.com.

This is an open-access article distributed under the terms of the Creative Commons Attribution (CC-BY) License, which permits unrestricted use, distribution, and reproduction in any medium, provided the original author and source are credited.

\section{REFERENCES}

1. World Health Organization, 2018. World Malaria Report 2018. Available at: https://www.who.int/malaria/publications/ world-malaria-report-2018/report/en/. Accessed March 28, 2019.

2. World Health Organization, 2015. Global Technical Strategy for Malaria 2016-2030. London, United Kingdom: WHO.
3. World Health Organization, Malaria Vaccine Funders Group, 2013. Malaria Vaccine Technology Roadmap 2013. Available at: https://www.malariavaccine.org/sites/www.malariavaccine.org/ files/content/page/files/TRM_update_nov13.pdf. Accessed February $18,2019$.

4. European Medicines Agency SMH, 2015. First Malaria Vaccine Receives Positive Scientific Opinion from EMA 2015. Available at: http://www.ema.europa.eu/ema/index.jsp?curl=pages/ news_and_events/news/2015/07/news_detail_002376.jsp\&mid= WC0b01ac058004d5c1. Accessed March 15, 2018.

5. World Health Organization, 2018. Malaria vaccine, 2016. WHO position paper-January 2016. Wkly Epidemiol Rec 91: 33-52.

6. World Health Organization, 2017. Ghana, Kenya and Malawi to Take Part in WHO Malaria Vaccine Pilot Programme. Available at: https://www.afro.who.int/news/ghana-kenya-and-malawitake-part-who-malaria-vaccine-pilot-programme. Accessed March 20, 2019.

7. Hay SI, Smith DL, Snow RW, 2008. Measuring malaria endemicity from intense to interrupted transmission. Lancet Infect Dis 8: 369-378.

8. Hay SI, Rogers DJ, Toomer JF, Snow RW, 2000. Annual Plasmodium falciparum entomological inoculation rates (EIR) across Africa: literature survey, internet access and review. Trans R Soc Trop Med Hyg 94: 113-127.

9. Drakeley $C$ et al., 2017. Longitudinal estimation of Plasmodium falciparum prevalence in relation to malaria prevention measures in six sub-Saharan African countries. Malar J 16: 433.

10. Smith DL, Guerra CA, Snow RW, Hay SI, 2007. Standardizing estimates of the Plasmodium falciparum parasite rate. Malar $\mathrm{J}$ 6: 131.

11. World Health Organization, 2017. Malaria Vaccine Implementation Programme (MVIP). Available at: https://www.who.int/immunization/ diseases/malaria/malaria_vaccine_implementation_programme/ about/en/. Accessed March 22, 2019.

12. World Health Organization, 2019. Malaria: Areas of Work - Diagnostic Testing. Available at: https://www.who.int/malaria/ areas/diagnosis/nucleic-acid-amplification-tests/en/. Accessed February 18, 2019.

13. Okell LC, Ghani AC, Lyons E, Drakeley CJ, 2009. Submicroscopic infection in Plasmodium falciparum-endemic populations: a systematic review and meta-analysis. J Infect Dis 200: 1509-1517.

14. Landis JR, Koch GG, 1977. The measurement of observer agreement for categorical data. Biometrics 33: 159-174.

15. The malERA Refresh Consultative Panel on Combination Interventions and Modelling, 2017. malERA: an updated research agenda for combination interventions and modelling in malaria elimination and eradication. PLoS Med 14: e1002453.

16. Diallo A et al., 2017. An epidemiological study to assess Plasmodium falciparum parasite prevalence and malaria control measures in Burkina Faso and Senegal. Malar J 16: 63.

17. Sylla K et al., 2015. Sero-epidemiological evaluation of Plasmodium falciparum malaria in Senegal. Malar J 14: 275.

18. Tine RCK et al., 2013. Parasitic infections among children under five years in Senegal: prevalence and effect on anaemia and nutritional status. ISRN Parasitol 2013: 272701

19. Ceesay SJ et al., 2008. Changes in malaria indices between 1999 and 2007 in The Gambia: a retrospective analysis. Lancet 372: 1545-1554.

20. Nankabirwa J, Brooker SJ, Clarke SE, Fernando D, Gitonga CW, Schellenberg D, Greenwood B, 2014. Malaria in school-age children in Africa: an increasingly important challenge. Trop Med Int Health 19: 1294-1309.

21. Felger I, Maire M, Bretscher MT, Falk N, Tiaden A, Sama W, Beck $\mathrm{H}-\mathrm{P}$, Owusu-Agyei S, Smith TA, 2012. The dynamics of natural Plasmodium falciparum infections. PLoS One 7: e45542.

22. Aron JL, 1983. Dynamics of acquired immunity boosted by exposure to infection. Math Biosci 64: 249-259.

23. Ceesay SJ et al., 2015. Malaria prevalence among young infants in different transmission settings, Africa. Emerg Infect Dis 21: 1114-1121.

24. Asua V, Tukwasibwe S, Conrad M, Walakira A, Nankabirwa JI, Mugenyi L, Kamya MR, Nsobya SL, Rosenthal PJ, 2017. Plasmodium species infecting children presenting with malaria in Uganda. Am J Trop Med Hyg 97: 753-757. 
25. Doctor SM et al., 2016. Low prevalence of Plasmodium malariae and Plasmodium ovale mono-infections among children in the Democratic Republic of the Congo: a population-based, crosssectional study. Malar J 15: 350.

26. Gneme A, Guelbeogo WM, Riehle MM, Tiono AB, Diarra A, Kabre GB, Sagnon N, Vernick KD, 2013. Plasmodium species occurrence, temporal distribution and interaction in a child-aged population in rural Burkina Faso. Malar J 12: 67.

27. Twohig KA, Pfeffer DA, Baird JK, Price RN, Zimmerman PA, Hay SI, Gething PW, Battle KE, Howes RE, 2019. Growing evidence of Plasmodium vivax across malaria-endemic Africa. PLoS Negl Trop Dis 13: e0007140.

28. Avicor SW, Wajidi MFF, Owusu EO, 2017. To coil or not to coil: application practices, perception and efficacy of mosquito coils in a malaria-endemic community in Ghana. Environ Sci Pollut Res Int 24: 21138-21145.

29. Hogarh JN, Agyekum TP, Bempah CK, Owusu-Ansah EDJ, Avicor SW, Awandare GA, Fobil JN, Obiri-Danso K, 2018. Environmental health risks and benefits of the use of mosquito coils as malaria prevention and control strategy. Malar J 17: 265.

30. Lukwa N, Chiwade T, 2008. Lack of insecticidal effect of mosquito coils containing either metofluthrin or esbiothrin on Anopheles gambiae sensu lato mosquitoes. Trop Biomed 25: 191-195.

31. World Health Organization, 2017. Malaria Policy Advisory Committee (MPAC) Meeting Report 2017. Geneva, Switzerland: WHO. Contract No.: WHO/HTM/GMP/MPAC/2017.21. 